\title{
Adaptive Shadow Compensation for Face Recognition Under Illumination Variations
}

\author{
Cheng-Ta Hsieh ${ }^{\mathrm{a}}$, Kae-Horng Huang ${ }^{\mathrm{b}}$, Chang-Hsing Lee ${ }^{\mathrm{b}^{*}}$, Chin-Chuan Han ${ }^{\mathrm{c}}$, Kuo-Chin Fan ${ }^{\mathrm{a}}$ \\ ${ }^{\mathrm{a}}$ Department of Computer Science \& Information Engineering, National Central University, Taoyuan, Taiwan \\ ${ }^{\mathrm{b}}$ Department of Computer Science \& Information Engineering, Chung Hua University, Hsinchu, Taiwan \\ ${ }^{\mathrm{c}}$ Department of Computer Science \& Information Engineering, National United University, Miaoli, Taiwan
}

*Corresponding Author: chlee@chu.edu.tw

\begin{abstract}
Robust face recognition under variant illumination conditions is a challenging research problem. In this paper, a new shadow compensation method, called adaptive shadow compensation (ASC), will be proposed to eliminate shadows in the face image due to non-frontal lighting directions. ASC performs shadow compensation in each local region, based on Fourier analysis on each local image block, to remove as much shadows as possible. Null-space linear discriminant analysis is then employed to extract discriminant features from ASC compensated images. Experimental results on the Yale B face database show that the proposed ASC method can achieve high face recognition accuracy under illumination variations.
\end{abstract}

Keywords: face recognition, shadow compensation, Fourier transform.

\section{Introduction}

Face recognition has a number of applications, including identity identification, access control, surveillance, human-computer interaction, etc. Many face recognition approaches have been proposed in the last few decades. Most methods can get high recognition accuracy in well controlled environments. However, the recognition accuracy will deteriorate greatly under different environmental conditions such as illumination, pose, and expression variations. Many methods were developed for face recognition under variant illumination conditions [1-3]. These methods can be classified into three categories: face modeling based approaches [4-6], invariant feature extraction approaches [3, 7-13], and image preprocessing techniques [14-21].
In the face modeling based approaches, a number of face images under varying lighting conditions was used to construct an illumination model such as illumination cone model [4], spherical harmonic model [5], 3D linear subspace [6], etc. One major problem with the face modeling approaches is that it needs a number of training images in the model learning process.

In the invariant feature extraction strategy, robust illumination invariant features were extracted for image matching. The most well-known invariant features include gradient face [3], local binary pattern (LBP) [7], 2D Gabor-like filters [8], phase spectrum [9-11], quotient image (QI) [12], self-quotient image (SQI) [13], etc.

In the image preprocessing techniques, some preprocessing/normalization methods were employed to normalize the face images such that the preprocessed images appear to be consistent even though they were taken under different illumination conditions. Several well-known image preprocessing methods, including histogram equalization (HE) [14, 15], Gamma correction [15], logarithm transform [16], retinex algorithm [17], DCT-compensation [18, 19], and DFT-compensation [20, 21], were widely used to adjust the image intensities such that the processed images will have similar appearance to those images taken in proper illumination conditions. Choi et al. [20] proposed the shadow compensation (SC) method, based on Fourier analysis of the input image, to solve face recognition problem under illumination variations. Their experimental results on face images having variant lighting conditions have shown that SC outperforms HE and other well-known approaches in terms of recognition accuracy.

In this paper, we will propose a novel shadow compensation method, called adaptive shadow compensation (ASC), for robust face recognition under 
different illumination conditions. The proposed ASC method performs shadow compensation in each local region, based on Fourier analysis, rather than on the whole image. Thus, local details can be well compensated. We will first review the SC method [20] in Section 2 and then describe the proposed ASC method in Section 3. Experimental results showing the effectiveness of the proposed ASC method will be shown in Section 4. Finally, a brief conclusion will be given in Section 5 .

\section{Review of Shadow Compensation Method}

Oppenheim et al. have shown that the phase information retains most of the intelligibility property of an image [22, 23]. Typically, the phase spectrum acts to the locations of edges in an image. Thus, the discriminant information will be kept in the phase spectrum of the image. Give a face image having shadow regions, the Fourier phase spectrum usually keeps unchanged while the Fourier magnitude spectrum will be changed greatly in those shadow areas. Therefore, the SC method proposed by Choi et al. [20] tries to compensate for the magnitude spectrum of an input image in order to get a recovered image having proper intensities in those shadow regions. To get the shadow compensated image, a set of reference images having proper illuminations were collected and transformed to the frequency domain by using 2D Discrete Fourier Transform (2D-DFT). The average of the magnitude spectra, notated by $\left|F^{a v g}(u, v)\right|$, of these reference images will be used for shadow compensation purpose. Given an input image, it is first transformed into frequency domain. Then, its magnitude spectrum $|F(u, v)|$ and the average magnitude spectrum $\left|F^{a v g}(u, v)\right|$ are combined to obtain the modified magnitude spectrum:

$$
\left|F^{M}(u, v)\right|=\frac{1}{2}|F(u, v)|+\frac{1}{2}\left|F^{a v g}(u, v)\right|
$$

The phase spectrum of the input image $\Phi(u, v)$ and the modified magnitude spectrum $\left|F^{M}(u, v)\right|$ will constitute the modified Fourier spectrum of the shadow compensated image:

$$
F^{M}(u, v)=\left|F^{M}(u, v)\right| e^{j \phi(u, v)}
$$

Thus, the recovered face image can be obtained by performing inverse 2D-DFT on the modified Fourier spectrum.

The SC method can generate a recovered image with some shadow regions being property compensated. However, there still exist some distorted regions in the recovered image (please Fig. 2(c)). This is due to the fact that the SC method modified the magnitude spectrum of the whole image, without considering the characteristics of different local regions, to reduce the effect of shadows. As a result, those local details cannot be properly compensated. In this paper, we will propose a novel shadow compensation method, called adaptive shadow compensation (ASC), for robust face recognition under variant illumination conditions. The proposed ASC method performs shadow compensation in each local region, based on Fourier analysis on each local image block, rather than on the whole image. Thus, intensity in local details can be well compensated.

\section{Adaptive Shadow Compensation (ASC) for Face Recognition}

The proposed ASC method, robust to face recognition under variant illumination conditions, tries to obtain a restored face image with each local region being well compensated. Fig. 1 shows the block diagram of the proposed ASC method. First, each face image $I(x, y)$ is enhanced using HE to get an enhanced image $I_{\mathrm{HE}}(x, y)$. The $\mathrm{HE}$ enhanced image will be decomposed into a number of image blocks, with each block of size $K \times K$ pixels. Shadow compensation using Fourier analysis will be conducted on each local image block such that shadows can be properly removed, in order to get a restored face image appearing as consistent with normal face images as possible.

\subsection{Adaptive Shadow Compensation}

Let $\boldsymbol{I}^{T}=\left\{I_{1}, I_{2}, \cdots, I_{L}\right\}$ denote the set of training images. Further, let $\boldsymbol{B}_{i, a, b}$ be the decomposed image block of size $K \times K$ at location $(a, b)$ of the $i$-th training image, $a=1$, $2, \ldots, M, b=1,2, \ldots, N$. Therefore, each image is decomposed into $M \times N$ image blocks. We then perform 2D-DFT on each image block to get the Fourier spectrum:

$$
\begin{array}{r}
F_{i, a, b}(u, v)=\frac{1}{K^{2}} \sum_{m=0}^{K-1} \sum_{n=0}^{K-1} B_{i, a, b}(u, v) e^{-j 2 \pi\left(\frac{u m}{K}+\frac{v n}{K}\right)}, \\
u=0,1, \cdots, K-1, v=0,1, \cdots, K-1
\end{array}
$$

Then, we can compute the average magnitude spectrum of the co-located image blocks of all training images:

$$
\begin{aligned}
& \left|F_{a, b}^{a v g}(u, v)\right|=\frac{1}{N} \sum_{i=1}^{L}\left|F_{i, a, b}(u, v)\right|, \\
& \quad u=0,1, \cdots, K-1, v=0,1, \cdots, K-1
\end{aligned}
$$




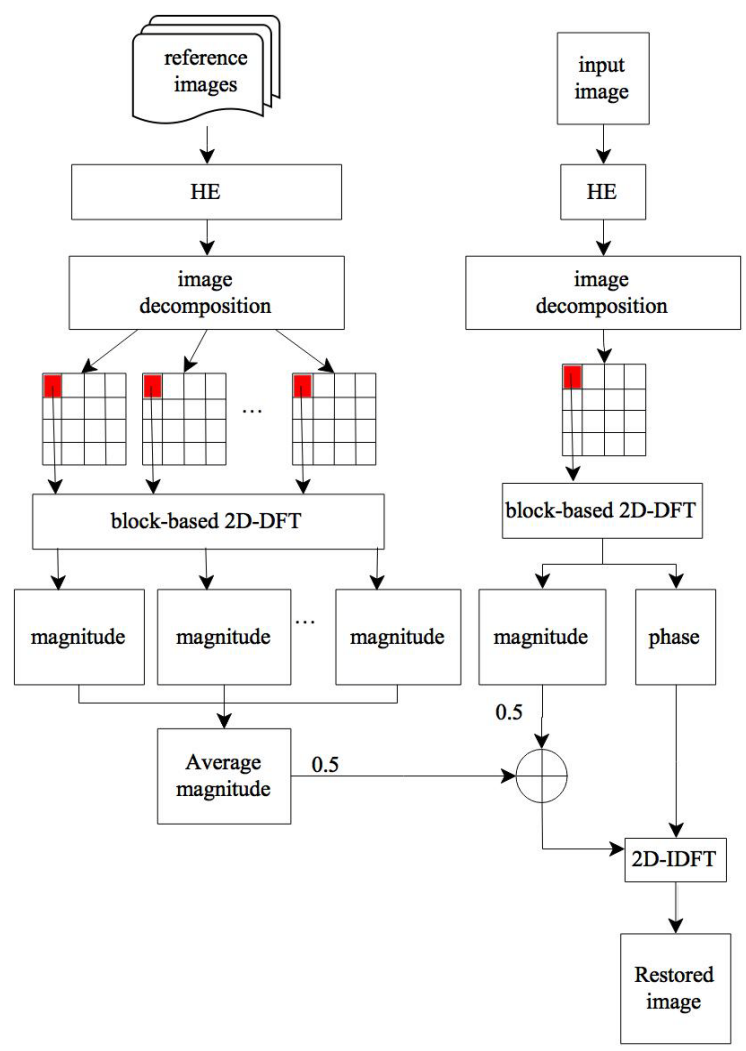

Fig. 1. Block diagram of the proposed ASC method

Similarly, for an input image, it is decomposed into a number of image blocks and each block is transformed using 2D-DFT to get the Fourier coefficients. Let $\left|F_{a, b}(u, v)\right|$ and $\Phi_{a, b}(u, v)$ denote respectively the magnitude spectrum and phase spectrum of the decomposed image block located at $(a, b)$. The modified magnitude spectrum of this image block can be obtained by

$$
\begin{aligned}
\left|F_{a, b}^{M}(u, v)\right|=\frac{1}{2}\left|F_{a, b}(u, v)\right|+\frac{1}{2}\left|F_{a, b}^{a v g}(u, v)\right|, \\
u=0,1, \cdots, K-1, v=0,1, \cdots, K-1
\end{aligned}
$$

Thus, the modified spectrum $F_{a, b}(u, v)$ can be derived from the modified magnitude spectrum $\left|F_{a, b}^{M}(u, v)\right|$ and the phase spectrum $\Phi_{a, b}(u, v)$ of the input image:

$$
\begin{aligned}
& F_{a, b}^{M}(u, v)=\left|F_{a, b}^{M}(u, v)\right| e^{j \phi_{a, b}(u, v)}, \\
& u=0,1, \cdots, K-1, v=0,1, \cdots, K-1
\end{aligned}
$$

Finally, each shadow compensated image block can be recovered by performing inverse 2D-DFT on the modified spectrum $F_{a, b}^{M}(u, v)$ :

$$
\begin{aligned}
I_{a, b}^{M}(m, n)= & \sum_{u=0}^{K-1} \sum_{v=0}^{K-1} F_{a, b}^{M}(u, v) e^{j 2 \pi\left(\frac{u m}{K}+\frac{v n}{K}\right)}, \\
& m=0,1, \cdots, K-1, n=0,1, \cdots, K-1
\end{aligned}
$$

Fig. 2 shows a number of images and the processed images using HE, SC, and ASC. From this figure, we can see that either HE or SC will produce an image with shadow regions being enhanced to some extent. However, noises in the shadow region are also visibly amplified. Overall, the proposed ASC method will generate a restored image with every local region being properly compensated, than HE or SC. Thus, it is expected that high recognition accuracy can be obtained if the ASC processed images were used for face recognition.

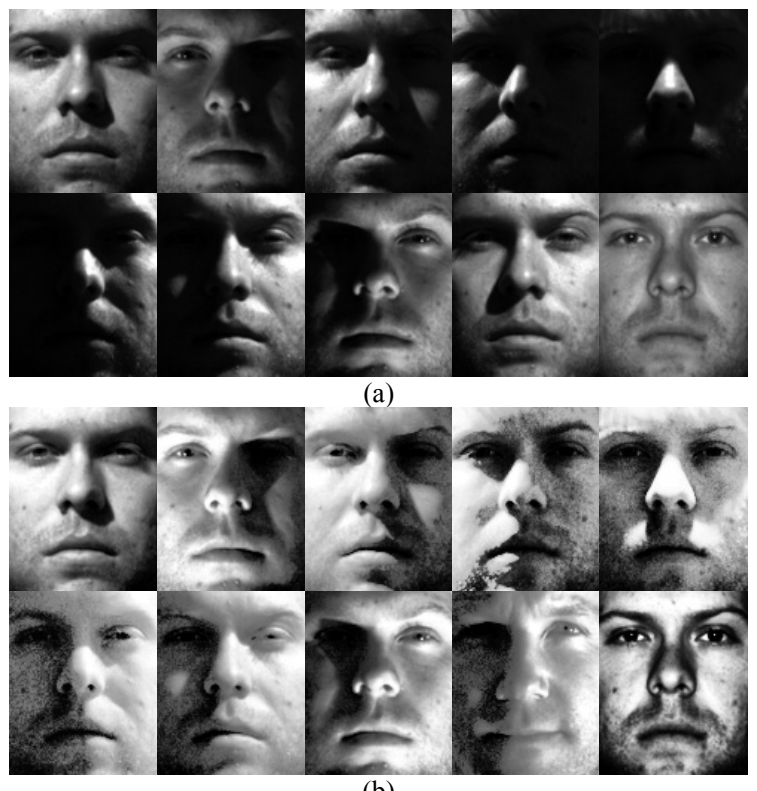

(b)

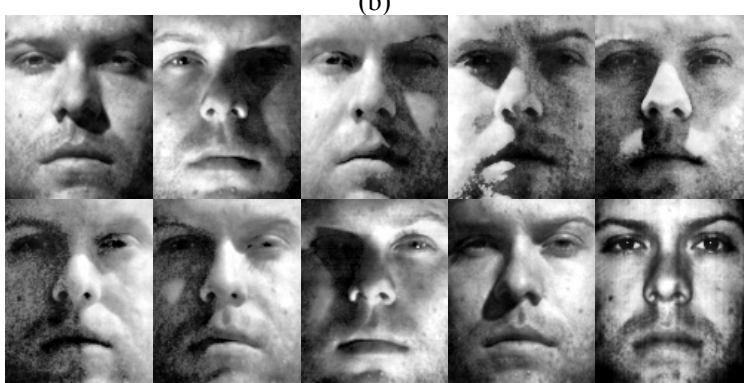

(c)

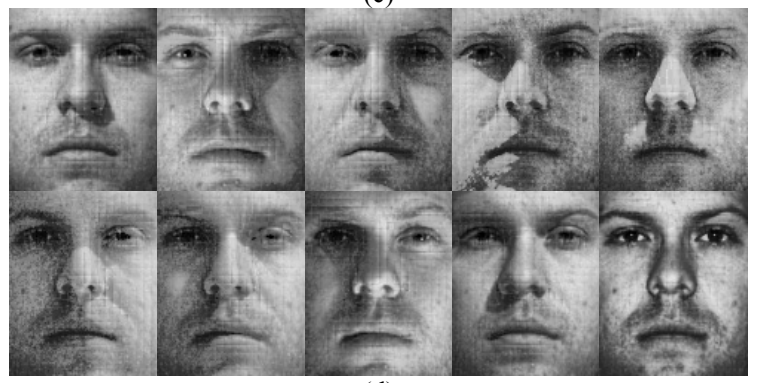

(d)

Fig. 2. Restored images using different preprocessing methods. (a) original images (b) enhanced images using HE (c) restored images using SC [20] (d) restored images using ASC. 


\subsection{Feature Extraction and Recognition}

Similar to the SC approach [20], the null space based linear discriminant analysis (NLDA) [24, 25] will be used to extract discriminant features from the restored images using ASC. In NLDA, the between-class scatter is maximized in the null space of the within-class scatter matrix. Assuming that the training set consists of $C$ classes, with $N_{i}$ samples in the $i$-th class. The between-class scatter matrix $\boldsymbol{S}_{b}$ and within-class scatter matrix $\boldsymbol{S}_{w}$ are defined as follows:

$$
\begin{gathered}
\boldsymbol{S}_{b}=\frac{1}{N} \sum_{i=1}^{C} N_{i}\left(\boldsymbol{\mu}^{i}-\boldsymbol{\mu}_{G}\right)\left(\boldsymbol{\mu}^{i}-\boldsymbol{\mu}_{G}\right)^{\mathrm{T}}, \\
\boldsymbol{S}_{w}=\sum_{i=1}^{C} \sum_{\mathbf{x} \in\left\{\boldsymbol{C}_{i}\right\}}\left(\mathbf{x}-\boldsymbol{\mu}^{i}\right)\left(\mathbf{x}-\boldsymbol{\mu}^{i}\right)^{\mathrm{T}},
\end{gathered}
$$

where $\mathbf{x}$ is a training image represented in a vector form, $\boldsymbol{\mu}_{G}$ is the mean vector of all training images, $\boldsymbol{\mu}^{i}$ is the mean vector of the training images in class $\boldsymbol{C}_{i}$. In general, the dimension of the image space is larger than the sample number in the training set. As a result, $\boldsymbol{S}_{w}$ will become singular and results in the so-called small sample size (SSS) problem. Since the null space of $\boldsymbol{S}_{w}$ contains much discriminative information, the objective function of NLDA is defined by

$$
\boldsymbol{W}_{\text {opt }}=\underset{\boldsymbol{W}:\left|\boldsymbol{W}^{\mathrm{T}} \boldsymbol{S}_{w} \boldsymbol{W}\right|=0}{\operatorname{argmin}}\left|\boldsymbol{W}^{\mathrm{T}} \boldsymbol{S}_{b} \boldsymbol{W}\right|
$$

$\boldsymbol{W}_{\text {opt }}$ is the transformation matrix consisting of the $d$ eigenvectors that maximizes the between-class scatter. This matrix will be used to project each image vector $\mathbf{x}$ to get a $d$-dimensional discriminant feature vector $\mathbf{y}$ :

$$
\mathbf{y}=\boldsymbol{W}_{o p t}^{\mathrm{T}} \mathbf{x}
$$

In the recognition process, the Euclidean distance is used to measure the distance between two discriminant feature vectors. Nearest neighbor rule will be employed to determine the recognition class of each input image.

\section{Experimental Results}

\subsection{The Face Database}

In this paper, The Yale B face database [4] is used to evaluate the performance of the proposed ASC method. The Yale B database consists of 10 individuals. The images of each individual have nine different poses and with 64 illuminations per pose. We used the face images in the front pose, which was divided into 5 subsets, for performance evaluation. The division of the image subsets depends on the angle between the direction of light source and the camera's axis. The light direction angles for these five subsets are $0^{\circ}-12^{\circ}, 12^{\circ}-25^{\circ}, 25^{\circ}-50^{\circ}, 50^{\circ}-77^{\circ}$, and $>77^{\circ}$ (please see Fig. 3). In this paper, the images belong to subset 1 , which have proper illuminations, were chosen as the training set and images in the other subsets were used as the testing set. These training images will be treated as the reference images for generating the average magnitude spectrum in ASC. For each image, the eyes are aligned to fixed positions and each image is rescaled to be size of $120 \times 100$ pixels.

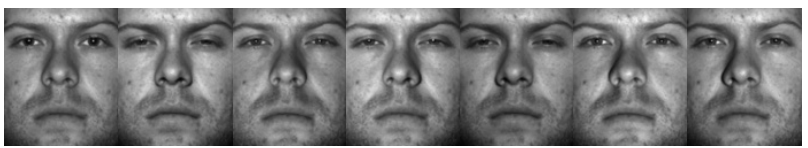

(a)

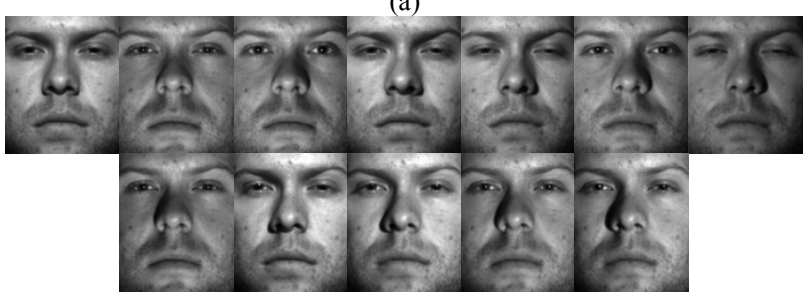

(b)

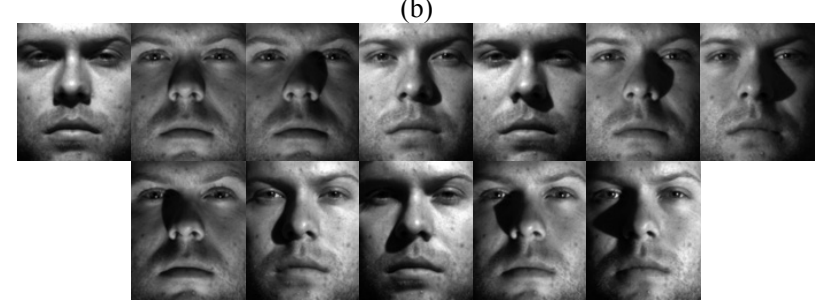

(c)
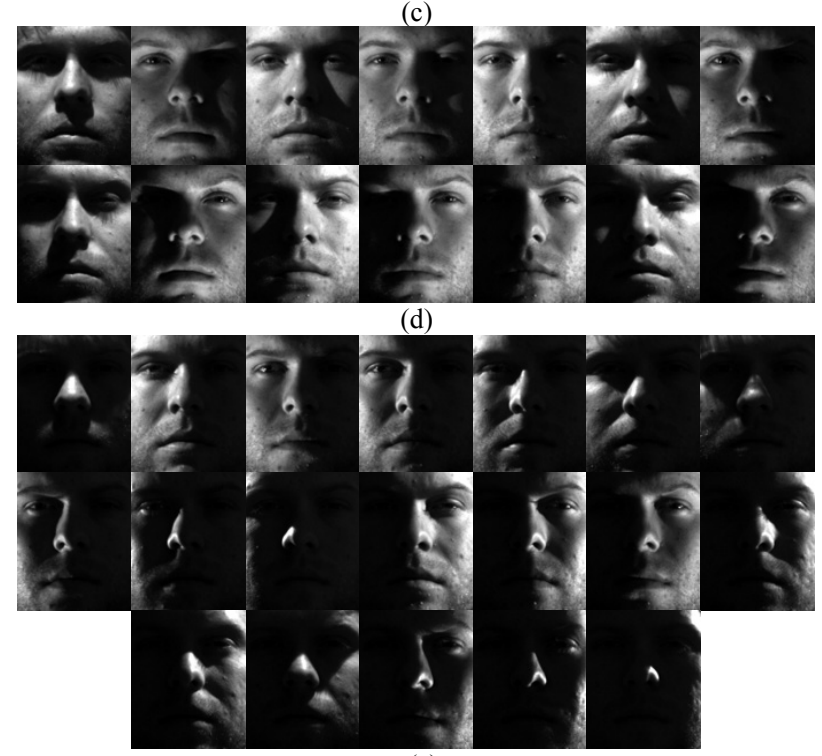

(e)

Fig. 3. An example showing images in each subset (a) subset 1 (b) subset 2 (c) subset 3 (d) subset 4 (e) subset 5 


\subsection{Comparison of Recognition Accuracy}

In the experiment, NLDA performed on the raw images $\left(\boldsymbol{I}_{R A W}\right)$, image enhanced using $\operatorname{HE~}\left(\boldsymbol{I}_{H E}\right)$, restored image using SC $\left(\boldsymbol{I}_{S C}\right)$, and restored images using ASC $\left(\boldsymbol{I}_{A S C}\right)$, were compared. Fig. 4 compares the recognition accuracy of these methods for different number of discriminant feature vector $(d)$. From this figure, we can see that SC outperforms HE. Further, the proposed ASC yields the best recognition accuracy.

Table 1 compares the recognition accuracy of each subset given that the feature dimension $d=9$. This table reveals that the proposed ASC method always yields the best recognition result for every subset. That is, ASC is more robust to illumination variations than $\mathrm{HE}$ or SC.

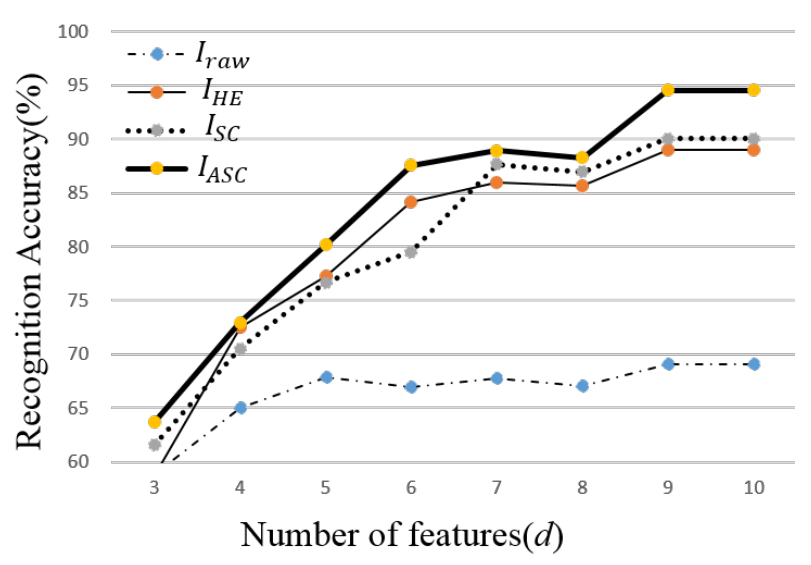

Fig. 4. Comparison of Recognition accuracy on the Yale B face database

Table 1. Recognition accuracy on the YALE B database (\%)

\begin{tabular}{|c|c|c|c|c|c|c|}
\hline Data & Subset 1 & Subset 2 & Subset 3 & Subset 4 & Subset 5 & All \\
\hline $\boldsymbol{I}_{R A W}$ & 100 & 100 & 95 & 37.8 & 12.6 & 69.1 \\
\hline $\boldsymbol{I}_{H E}$ & 100 & 100 & 100 & 73.5 & 71.5 & 89.0 \\
\hline $\boldsymbol{I}_{S C}$ & 100 & 100 & 100 & 75.0 & 75.2 & 90.0 \\
\hline $\boldsymbol{I}_{A S C}$ & $\mathbf{1 0 0}$ & $\mathbf{1 0 0}$ & $\mathbf{1 0 0}$ & $\mathbf{8 8 . 5}$ & $\mathbf{8 4 . 2}$ & $\mathbf{9 4 . 5}$ \\
\hline
\end{tabular}

\section{Conclusions}

In this paper, a new shadow compensation method, called adaptive shadow compensation (ASC), is proposed to eliminate shadows in the face image due to non-frontal lighting directions. ASC performs shadow compensation in each local image block, based on Fourier analysis, to remove as much shadows as possible. Null-space linear discriminant analysis is then employed to extract discriminant features from ASC compensated images. Experiments performed on the Yale B face database have shown that the proposed ASC method can reserve more detailed information in shadow regions, and thus can yield high face recognition accuracy. In the near future, we will try to apply the proposed ASC method to compensate for over-exposed, under-exposed, or dark images.

\section{Acknowledgment}

This research was supported in part by the Ministry of Science and Technology of R.O.C. under contract MOST 102-2632-E-216-001-MY3, MOST 104-2221-E-216-010MY2, and MOST 104-2221-E-216-008.

\section{References}

[1] X. Zou, J. Kittler, and K. Messer, "Illumination Invariant Face Recognition: A Survey," in Proceedings of first IEEE conference Biometric: Theory, Application and System, 2007.

[2] R. Javier and Q. Julio, "Illumination Compensation and Normalization in Eigenspace-based Face Recognition: A Comparative Study of Different Pre-processing Approaches," Pattern Recognition Letters, vol. 29, no. 14, pp. 1966-1979, 2008.

[3] T. Zhang, Y. Y. Tang, B. Fang, Z. Shang, and X. Liu, "Face Recognition Under Varying Illumination Using Gradientfaces," IEEE Transactions on Image Processing, vol. 18, pp. 2599-2606, 2009.

[4] A. S. Georghiades and P. N. Belhumeur, "From Few to Many: Illumination Cones Models for Face Recognition Under Variable Lighting and Pose," IEEE Transactions on Pattern Analysis and Machine Intelligence, vol. 23, no. 2, pp. 643-660, 2001.

[5] R. Ramamoorthi, "Analytic PCA Construction for Theoretical Analysis of Lighting Variability, Including Attached Shadows, in a Single Image of Convex Lambertian Object," IEEE Transactions on Pattern Analysis and Machine Intelligence, vol. 24, pp. 1322-1333, 2002.

[6] P. N. Belhumeur, J. P. Hespanha, and D. J. Kriegman, "Eigenfaces vs. Fisherfaces: Recognition Using Class Specific Linear Projection," IEEE Transactions on Pattern Analysis and Machine Intelligence, vol. 19, no. 7, pp.711-720, 1997.

[7] T. Ahonen, A. Hadid, and M. Pietikäinen, "Face Description with Local Binary Patterns: Application to 
Face Recognition," IEEE Transactions on Pattern Analysis and Machine Intelligence, vol. 28, no. 12, pp. 2037-2041, 2006.

[8] Y. Adini, Y. Moses, and S. Ullman, "Face recognition: The Problem of Compensating for Changes in Illumination Direction," IEEE Transactions on Pattern Analysis and Machine Intelligence, vol. 19, pp. 721-732, 1997.

[9] M. Savvides, B. V. K. VijayaKumar, and P. K. Khosla, "Eigenphases vs. Eigenfaces," International Conference on Pattern Recognition, pp. 810-813, 2004.

[10] J. Heo, M. Savvides, and B. V. K. Vijayakumar, "Illumination Tolerant Face Recognition Using Phase-only Support Vector Machines in the Frequency Domain," International Conference on Advances in Pattern Recognition, pp. 66-73, 2005.

[11] X. Y. Jing, Y. Y. Tang, and D. Zhang, “A Fourier-LDA Approach for Image Recognition," Pattern Recognition Letters, vol. 38, no. 4, pp. 453-457.

[12] A. Shashua and T. Riklin-Raviv, "The Quotient Image: Class Based Re-rendering and Recognition with Varying Illuminations," IEEE Transactions on Pattern Analysis and Machine Intelligence, vol. 23, no. 2, pp. 129-139, 2001

[13] H. Wang, S. Li, and Y. Wang, "Face Recognition Under Varying Lighting Conditions Using Self Quotient Image," IEEE International Conference on Automatic Face and Gesture Recognition, pp. 819-824, 2004.

[14] S. M. Pizer and E. P. Amburn, "Adaptive Histogram Equalization and Its Variations," Computer Vision, Graph, and Image Process, vol. 39, no. 3, pp. 355-368, 1987.

[15] S. Shan, W. Gao, B. Cao, and D. Zhao, "Illumination Normalization for Robust Face Recognition Against Varying Lighting Conditions," IEEE International Workshop on Analysis and Modeling of Faces and Gestures, 2003.

[16] M. Savvides and V. Kumar, "Illumination Normalization Using Logarithm Transforms for Face Authentication," in Proceedings of International Association for Pattern Recognition, Audio and Video Based Biometric Person Authentication. pp. 549-556. 2003.

[17] L. Tang, S. Chen, W. Liu and Y. Li, "Improved Retinex Image Enhancement Algorithm," Conference on Challenges in Environmental Science and Computer
Engineering, 2011.

[18] W. Chen, M. J. Er, and S. Wu, "Illumination Compensation and Normalization for Robust Face Recognition Using Discrete Cosine Transform in Logarithmic Domain," IEEE Transactions on System, Man, Cybernetic, Part B, vol. 36, no. 2, pp. 458-466, 2006.

[19] A. Abbas, M. I. Khalil, S. Abdel-Hay, and H. M. Fahmy, "Expression and Illumination Invariant Preprocessing Technique for Face Recognition," International Conference on Computer Engineering \& Systems, 2008.

[20] S. I. Choi and G. M. Jeong, "Shadow Compensation Using Fourier Analysis with Application to Face Recognition," IEEE Signal Processing Letters, vol. 18, no. $1,2011$.

[21] H. Kaur and A. Kaur, "Illumination Invariant Face Recognition," International Journal of Computer Application, vol. 64, no. 21, pp. 23-27, 2013.

[22] A. V. Oppenheim and J. S. Lim, "The Important of Phase in Signals," Proceedings of the IEEE, vol. 69, no. 5, pp. 529-541, 1981.

[23] M. H. Hayes, J. S. Lin and A. V. Oppenheim, "Signal Reconstruction from Phase or Magnitude," IEEE Transactions on Acoustic, Speech and Signal Processing, vol. ASSp-28, pp. 672-680, 1980.

[24] H. Cevikalp, M. Neamtu, M. Wilkes, and A. Barkana, "Discriminative Common Vectors for Face Recognition," IEEE Transactions on Pattern Analysis and Machine Intelligence, vol. 27, no. 1, pp. 4-13, 2005.

[25] L. F. Chen, H. Y. M. Liao, M. T. Ko, J. C. Lin and G. J. $\mathrm{Yu}$, "A New LDA-based Face Recognition System Which Can Solve the Small Sample Size Problem," Pattern Recognition, vol. 33, pp. 1713-1726, 2000. 